\title{
A few thoughts on "Interventional radiology in the management of benign biliary stenoses, biliary leaks and fistulas: a pictorial review"
}

\author{
Giovanni Mauri • Luca Maria Sconfienza
}

Received: 13 December 2012 / Accepted: 20 December 2012 / Published online: 30 January 2013

(C) The Author(s) 2013. This article is published with open access at Springerlink.com

\section{Dear Editor,}

We read with great interest the article by Krokidis and colleagues [1] recently published in Insights into Imaging. In this well-presented pictorial review, the authors point out the crucial role of interventional radiology as a minimally invasive approach to treat benign strictures, to divert the bile away from the site of the fistula, and to seal the leaking tract.

Balloon dilation was reported as the strategy of choice in the percutaneous treatment of benign biliary strictures (although burdened by $29-58 \%$ re-stenosis), while the role of stent placement was reported to still be controversial. A different approach can be the use of novel bioabsorbable biliary stents [2], which may limit the number of balloon dilations in bilioplasty, also overcoming the problem of stent withdrawal.

Regarding bile leaks, the authors discuss - inter alia - the role of percutaneous drainage of biliary collections, of coil/ gelfoam embolisation of bilio-vascular fistulas, and of covered stents. However, we underline that the most commonly used technique for treating postsurgical biliary leak is percutaneous transhepatic biliary drainage, which allow for bile diversion from the site of the fistula [3]. Moreover, an occlusion balloon placed above the site of the fistula has been demonstrated to allow for complete external drainage of the bile, thus representing a valuable option to treat postsurgical biliary leaks $[3,4]$.

\section{G. Mauri $\cdot$ L. M. Sconfienza $(\bowtie)$}

Servizio di Radiologia, IRCCS Policlinico San Donato,

Piazza Malan 2,

20097 San Donato Milanese, Milano, Italy

e-mail: io@lucasconfienza.it

\section{M. Sconfienza}

Dipartimento di Scienze Biomediche per la Salute, Università degli Studi di Milano, Piazza Malan 2,

20097 San Donato Milanese, Milano, Italy
In case of direct communication between a collection and the biliary system, it may happen that percutaneous drainage is not enough to allow for full healing and surgery may be required, as the authors show in Figure 3 [1]. We note that, in this particular setting, percutaneous cyanoacrilyc glue embolisation has been reported as a feasible technique, which may lead to fistula sealing, avoiding the morbidity related to surgical re-intervention [5].

Open Access This article is distributed under the terms of the Creative Commons Attribution License which permits any use, distribution, and reproduction in any medium, provided the original author(s) and the source are credited.

\section{References}

1. Krokidis M, Orgera G, Rossi M, Matteoli M, Hatzidakis A (2012) Interventional radiology in the management of benign biliary stenoses, biliary leaks and fistulas: a pictorial review. Insights Imaging. doi:10.1007/s13244-012-0200-1

2. Pedicini V, Poretti D, Tramarin M, Mauri G, Lanza E, Melchiorre F, Brambilla G, Cornalba G (2012) A novel bioabsorbable biliary stent in the percutaneous treatment of benign biliary stricture: preliminary experience. Abstract presented at Cardiovascular and Interventional Radiological Society of Europe (CIRSE)

3. Pedicini V, Poretti D, Mauri G, Trimboli RM, Brambilla G, Sconfienza LM, Cornalba GP, Sardanelli F (2010) Management of post-surgical biliary leakage with percutaneous transhepatic biliary drainage (PTBD) and occlusion balloon (OB) in patients without dilatation of the biliary tree: preliminary results. Eur Radiol 20:1061-1068

4. Cozzaglio L, Cimino M, Mauri G, Ardito A, Pedicini V, Poretti D, Brambilla G, Sacchi M, Melis A, Doci R (2011) Percutaneous transhepatic biliary drainage and occlusion balloon in the management of duodenal stump fistula. J Gastrointest Surg 15:1977-1981. doi:10.1007/s11605-011-1668-6

5. Mauri G, Sconfienza LM, Fiore B, Brambilla G, Pedicini V, Poretti D, Lutman RF, Montorsi M, Sardanelli F (2013) Post-surgical enteric fistula treatment with image-guided percutaneous injection of cyanoacrylic glue. Clin Radiol 68:59-63. doi:10.1016/ j.crad.2012.04.004 